\title{
THE RIGHT-POLARIZED NEUTRAL (ANTI)LEPTONS IN THE SOLAR NEUTRINO FLUX
}

\author{
Romanov Yu.I. \\ Moscow State University of Design and Technology, Russia \\ E-mail: romanov.yu.i@mail.ru
}

In the present work, a development [1], the flavor structure of solar neutrino is investigated.

The possibility of changing the flavor of solar electron neutrinos $v_{\mathrm{e}}$ is directly related to their helical properties. If the helicity changes (scenario of magnetic reorientations), within the left-polarized neutrino concept the "solar messengers" can be transformed into a right-polarized neutral particles, i.e. into an antineutrino of second and third generations $\left(\widetilde{v}_{\mu}^{R}\right.$ and $\left.\widetilde{v}_{\tau}^{R}\right)$. Part of the neutrino flow from the Sun is likely to undergo conversion also into related antineutrino $\widetilde{v}_{e}$. At the same time, according to the four-component theory, a change of the helicity leads to the conversion of solar neutrinos into a muon (right-polarized) neutrinos $v_{\mu}$. Consequently, it is possible, that if the helicity changes, the solar neutrinos can reach an electron target in the $\left\{v_{e}^{L}, \widetilde{v}_{e}^{R}, \widetilde{v}_{\mu}^{R}, \widetilde{v}_{\tau}^{R}\right\}$ or $\left\{v_{e}^{L}, \widetilde{v}_{e}^{R}, v_{\mu}^{R}, \widetilde{v}_{\tau}^{R}\right\}$ states of the wave (anti)neutrino packet.

The electroweak (SM) spectrum of recoil electrons containing admixture muon and tau components is obtained and analyzed for the scattering of monoenergetic beryllium solar neutrinos with energy of $0.862 \mathrm{MeV}$ on an electron target. These components correspond to the above predictions for neutrino states with contents of neutral leptons in the ratios given by the total SM-cross sections with threshold of $0.25 \mathrm{MeV}$ from final electrons.

The results of quantitative analysis and graphical image of the cross sections are presented in the form of tables and figures. For sake of comparison, all the figures show curve which characterized the successful (i.e. with flavor conservation) arrival of solar electron neutrinos on the Earth.

1. Yu.I.Romanov // Bull. Russian Acad. Sci. Physics. 2012. V.76. No.4. P.507. 